\title{
Long-term survival in a patient with acute promyelocytic leukemia with isolated meningeal relapse
}

\author{
Se Hyung Kim, Jina Yun, Hyun Jung Kim, Chan Kyu Kim, Sung Kyu Park, Dae Sik Hong \\ Department of Hematology-Oncology, Soonchunhyang University College of Medicine, Bucheon, Korea
}

p-ISSN 1738-7949 / e-ISSN 2092-9129

DOI: $10.5045 / \mathrm{kjh} .2010 .45 .3 .208$

Korean J Hematol 2010;45:208-10.

Received on March 1, 2010

Revised on August 3, 2010

Accepted on September 2, 2010

\section{Correspondence to}

Dae Sik Hong, M.D. Ph.D.

Department of Hematology-Oncology, Soonchunhyang University College of

Medicine, 1174, Joong-dong, Wonmi-gu,

Bucheon 420-767, Korea

Tel: $+82-32-621-6608$

E-mail: dshong@schbc.mc.kr

(C) 2010 Korean Society of Hematology
The meningeal involvement is rare in acute promyelocytic leukemia. We experienced a 39-year-old woman who achieved complete remission with all-trans retinoic acid, idarubicin and cytarabine therapy. Several months later, she complained of non-specific headache. Her complete blood cell count was normal, but magnetic resonance image of brain revealed focal meningeal enhancement and cerebrospinal fluid showed leukemic promyelocytes and $P M L / R A R A$ rearrangement. Bone marrow study showed hematologic, cytogenetic and molecular remission. She was treated with intrathecal and systemic chemotherapy and whole brain radiotherapy. The patient has survived for 68 months since the last systemic chemotherapy.

Key Words APL, Extramedullary involvement, CNS

\section{INTRODUCTION}

Acute promyelocytic leukemia (APL) is a specific type of acute myeloid leukemia (AML) characterized by the presence of abnormal promyelocytes in bone marrow and peripheral blood, young age at presentation, specific chromosomal abnormalities, coagulopathy, and a unique response to treatment with retinoic acid. Until recently, combination of all-trans retinoic acid (ATRA) and chemotherapy with cytarabine and anthracyclines has been the standard treatment for APL. Although treatment with ATRA has improved the outcome of APL, early bleeding due to coagulopathy and relapse are the main causes of death in patients with APL [1].

APL may relapse in central nervous system (CNS). However, CNS involvement at relapse is rare and is generally associated with a poor prognosis. We present a case of long-term survival in an APL patient who had achieved hematologic and molecular complete remission (CR) after treatment of meningeal relapse using ATRA, idarubicin and cytarabine.

\section{CASE REPORT}

A 39-year-old woman presented with headache and petechiae. Her complete blood count (CBC) showed a white blood cell (WBC) count of $8,600 / \mu \mathrm{L}$ consisting of $75 \%$ blasts, hemoglobin level of $9.4 \mathrm{~g} / \mathrm{dL}$, and platelet count of $13,000 / \mu \mathrm{L}$. Coagulation test showed prothrombin time of $13.5 \mathrm{sec}$, partial thromboplastin time of $26.1 \mathrm{sec}$, and fibrinogen level of $80 \mathrm{mg} / \mathrm{dL}$. APL was diagnosed on the basis of findings from bone marrow (BM) examination, and was confirmed by chromosomal analysis, which indicated the presence of $t(15 ; 17)$ (q22;q11.2) and molecular analysis, which indicated the presence of promyelocytic leukemia $(P M L) /$ retinoic acid receptor-a $(R A R A)$ rearrangement. Induction chemotherapy with idarubicin (intravenous administration of idarubicin $12 \mathrm{mg} / \mathrm{m}^{2} /$ day on days $2,4,6$, and 8) and ATRA (45 mg/m²) was administered to achieve CR. BM examination revealed normal karyotype and absence of the $P M L / R A R A$ rearrangement. The patient received consolidation therapy with 2 consecutive courses of idarubicin and 1 course of mitoxantrone, followed by maintenance therapy with ATRA (45 $\mathrm{mg} / \mathrm{m}^{2}$ on days $1-15$, every other month for 2 years).

After 17 months, the patient complained of non-specific headache for 1 month. Her physical examination was un-

\footnotetext{
This is an Open Access article distributed under the terms of the Creative Commons Attribution Non-Commercial License (http://creativecommons.org/licenses/by-nc/3.0)
} which permits unrestricted non-commercial use, distribution, and reproduction in any medium, provided the original work is properly cited. 


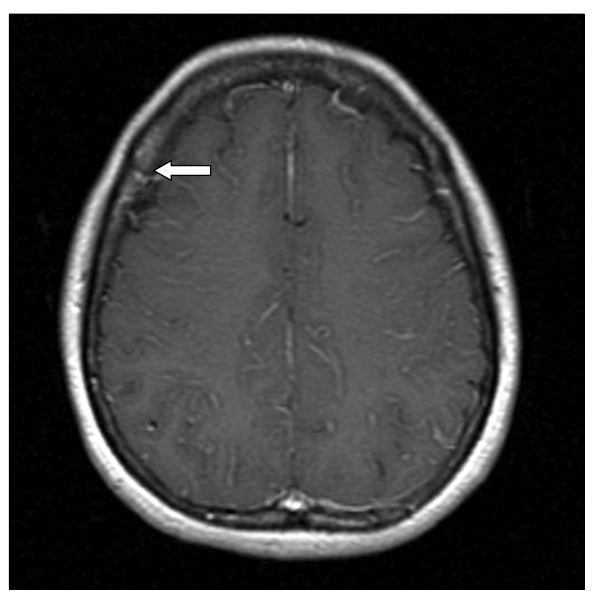

Fig. 1. Enhanced T1-weighted magnetic resonance image showing focal meningeal enhancement (arrow) in the right parietal lobe.

remarkable and her CBC was normal. Enhanced T1-weighted magnetic resonance image showed focal meningeal enhancement in the right parietal lobe (Fig. 1). Cerebrospinal fluid (CSF) microscopic finding showed numerous leukemic promyelocytes-containing prominent nuclear irregularities and variably granulated cytoplasm (Fig. 2) and $P M L / R A R A$ rearrangement was detected. BM study showed hematologic, cytogenetic, and molecular CR. The patient was diagnosed with meningeal relapse of APL and treated with intrathecal administration of methotrexate and cytarabine (methotrexate $12 \mathrm{mg}$ and cytarabine $50 \mathrm{mg}$ administered 3 times a week until the WBC count in the CSF reached zero), followed by whole-brain radiotherapy and systemic chemotherapy (cytarabine $2.0 \mathrm{~g} / \mathrm{m}^{2}$ I.V. b.i.d. on days $1-3$, etoposide 70 $\mathrm{mg} / \mathrm{m}^{2} \mathrm{I}$.V. on days $\left.1-4\right)$. The patient has survived for 68 months since the last systemic chemotherapy.

\section{DISCUSSION}

Extramedullary involvement at relapse is rarely observed in patients with APL. Skin, CNS, middle ear, lung, lymph node, mediastinum, thymus, spine, breast, pelvis, and gingival are the common extramedullary sites. The skin is the most common extramedullary site [2].

The symptoms of CNS involvement in APL are headache, vertigo, nausea, vomiting, visual disturbance, motor weakness, and seizure. In our case, the patient complained of non-specific headache. Although meningeal infiltration of leukemia is rare, CSF should be examined when symptoms of suspected CNS involvement are present. Interestingly, in our patient, CNS relapse did not coincide with hematologic and molecular relapse. Among the reported cases of APL, 6 continued to show hematologic and molecular remission despite CNS relapse [3-7].

ATRA induces differentiation of abnormal promyelocytes into mature granulocytes and induces CR in $80-90 \%$ of newly

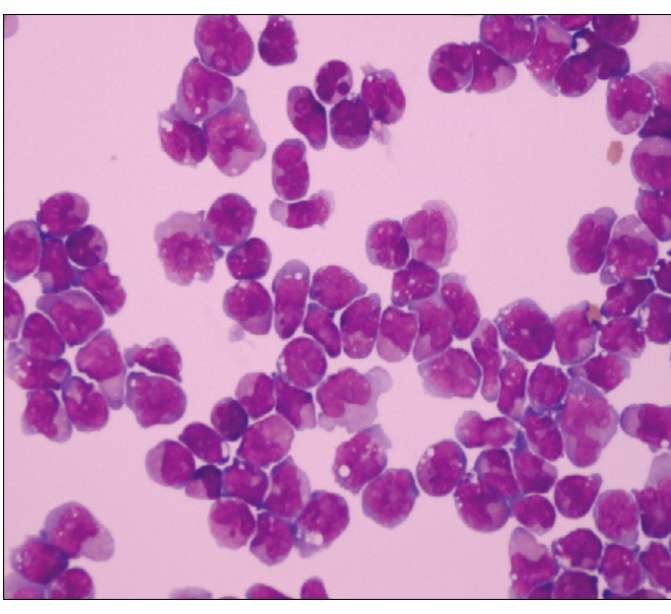

Fig. 2. Microscopic finding of cerebrospinal fluid showing numerous leukemic promyelocytes with prominent nuclear irregularities and variably granulated cytoplasms (Wright stain; $\times 1,000)$.

diagnosed and first-relapsed cases of APL [1, 8, 9]. After the advent of ATRA treatment, an increasing number of APL cases with the involvement of extramedullary sites at relapse have been reported. Thus, ATRA treatment may predispose APL patients to extramedullary relapse. Several biologic effects such as ATRA-mediated increase in the expression of certain adhesion molecules (e.g., CD11c, CD13, and CD56) have been suggested to play a role in extramedullary involvement of leukemic cells [10, 11]. A GIMEMA study compared the relapse pattern in APL patients receiving different treatments (ATRA+chemotherapy group vs. chemotherapy alone). Unlike patients receiving chemotherapy alone, patients receiving ATRA and chemotherapy did not have an increased risk of involvement of extramedullary sites at relapse. However, patients receiving ATRA had a higher prevalence of CNS involvement. These findings suggest that with the advent of ATRA and subsequent combination chemotherapy, a substantially higher fraction of patients who achieve long-term remission are at the risk of developing CNS relapse than patients who were treated with chemotherapy alone in the past [12].

The prognosis of CNS relapse in APL is generally poor. In particular, patients with multifocal CNS involvement had short survival time $[3,8]$. Our findings indicate that after multimodal treatment, patients with focal involvement of the CNS have longer survival times than patients with multifocal involvement of the CNS. Treatment of CNS relapse consists of intrathecal chemotherapy, radiation therapy, systemic chemotherapy with high-dose cytarabine, and treatment with agents such as ATRA. Although ATRA does not cross the blood-brain-barrier (BBB), it induces the differentiation of APL blasts present in the CSF of patients with CNS relapse [13]. High-dose cytarabine and arsenic trioxide $\left(\mathrm{AS}_{2} \mathrm{O}_{3}\right)$ pass through the $\mathrm{BBB}$ and could be effective for APL patients with CNS relapse [14, 15].

In summary, we presente a case of long-term survival in an APL patient with isolated meningeal relapse. CNS 
is the possible site of relapse in patients with CR after initial treatment. Therefore, precise evaluation and diagnosis by using approaches such as lumbar puncture and CSF examination should be performed, if CNS symptoms occur in patients with APL.

\section{REFERENCES}

1. Fenaux $\mathrm{P}$, Chastang $\mathrm{C}$, Chevret $\mathrm{S}$, et al. A randomized comparison of all transretinoic acid (ATRA) followed by chemotherapy and ATRA plus chemotherapy and the role of maintenance therapy in newly diagnosed acute promyelocytic leukemia. Blood 1999; 94:1192-200.

2. Wiernik PH, De Bellis R, Muxi P, Dutcher JP. Extramedullary acute promyelocytic leukemia. Cancer 1996;78:2510-4.

3. Bae SH, Ryoo HM, Cho HS, Lee JL, Lee KH, Hyun MS. Meningeal relapse in a Patient with acute promyelocytic leukemia: a case report and review of the literature. J Korean Med Sci 2004;19:311-4.

4. Anguita E, Villegas A, González FA, Díaz-Mediavilla J, Lo Coco F. Acute promyelocytic leukemia relapse in the central nervous system during hematologic and molecular remission. Am J Hematol 1999;60:170-1.

5. Mesa JR, Espinosa E, Losada R, Hernandez C, Martinez G, Hernandez P. Parotid and central nervous system relapse during complete hematologic remission in acute promyelocytic leukemia. Haematologica 1999;84:565-6.

6. Liso V, Specchia G, Pogliani EM, et al. Extramedullary involvement in patients with acute promyelocytic leukemia: a report of seven cases. Cancer 1998;83:1522-8.

7. Akoz AG, Dagdas S, Ozet G, Ceran F, Yilmaz M. Isolated central nervous system relapse during cytologic and molecular hematologic remission in two patients with acute promyelocytic leukemia. Hematology 2007;12:419-22.

8. Ko BS, Tang JL, Chen YC, et al. Extramedullary relapse after all-trans retinoic acid treatment in acute promyelocytic leukemia-the occurrence of retinoic acid syndrome is a risk factor. Leukemia 1999;13:1406-8.

9. Martino B, Vincelli I, Marino A, Comis M, Ronco F, Nobile F. Meningeal relapse in a patient with acute promyelocytic leukaemia treated with all-trans retinoic acid. Br J Haematol 1998;100:606-7.

10. Marchetti M, Falanga A, Giovanelli S, Oldani E, Barbui T. All-trans retinoic acid increases adhesion to endothelium of the human promyelocytic leukaemia cell line NB4. Br J Haematol 1996;93:360-6.

11. Saiki I, Fujii H, Yoneda J, et al. Role of aminopeptidase N (CD13) in tumor-cell invasion and extracellular matrix degradation. Int J Cancer 1993;54:137-43.

12. Specchia G, Lo Coco F, Vignetti M, et al. Extramedullary involvement at relapse in acute promyelocytic leukemia patients treated or not with all-trans retinoic acid: a report by the Gruppo Italiano Malattie Ematologiche dell'Adulto. J Clin Oncol 2001;19:4023-8.

13. Wiernik PH, De Bellis R, Muxi P, Dutcher JP. Extramedullary acute promyelocytic leukemia. Cancer 1996;78:2510-4.

14. Blaney SM, Balis FM, Poplack DG. Current pharmacological treatment approaches to central nervous system leukaemia. Drugs 1991;41:702-16

15. Au WY, Tam S, Fong BM, Kwong YL. Determinants of cerebrospinal fluid arsenic concentration in patients with acute promyelocytic leukemia on oral arsenic trioxide therapy. Blood 2008;112:3587-90. 